\title{
AERAÇÃO DE DISPOSITIVOS MÉDICOS ESTERILIZADOS A ÓXIDO DE ETILENO: CONSIDERAÇÕES ACERCA DA REGULAÇÃO BRASILEIRA
}

\author{
Aecization of medical devices sterilized to ethylene oxide: \\ considerations about Brazilian regulation
Aireación de dispositivos médicos esterilizados con óxido de etileno: consideraciones sobre la regulación brasileña \\ Eliana Auxiliadora Magalhães Costa ${ }^{1 *}$
}

\begin{abstract}
RESUMO: Objetivos: Descrever níveis residuais aceitáveis de óxido de etileno em dispositivos médicos, analisar processos de aeração recomendados e compará-los com a regulação brasileira. Método: Revisão integrativa da literatura, com descritores específicos, sem restrição de ano de publicação. Busca dos dados entre outubro e novembro de 2019 , que resultou em 34 estudos incluídos no estudo. Resultados: A regulação brasileira vigente está desatualizada em relação à classificação de produtos, à determinação de valores de resíduos tóxicos de óxido de etileno em dispositivos médicos e aos processos recomendados para a aeração desses produtos, podendo contribuir para riscos de eventos adversos para pacientes usuários de dispositivos inadequadamente aerados, e, consequentemente, urge sua atualização. Conclusão: As lacunas desse marco regulatório beneficiam indiretamente as empresas que terceirizam a esterilização a óxido de etileno ao omitir controles essenciais para a segurança do paciente exposto a possíveis resíduos tóxicos de óxido de etileno, favorecer práticas inseguras de esterilização de produtos para saúde, além de dificultar o controle de serviço de saúde pelas vigilâncias sanitárias do país. Palavras-chave: Equipamentos e provisões. Esterilização. Óxido de etileno. Resíduos tóxicos.
\end{abstract}

ABSTRACT: Objectives: To describe acceptable residual levels of ethylene oxide in medical devices, analyze recommended aesther processes and compare them with the Brazilian regulation. Method: Integrative literature review, with specific descriptors, without year of publication restriction. Data search between October and November 2019, which resulted in 34 studies included in the study. Results: Current Brazilian regulation is outdated in relation to product classification, the determination of waste values ethylene oxide toxic in medical devices and the recommended processes for the aecization of these products, and may contribute to risks of adverse events for patients users of inappropriately aenated devices, and consequently urge their update. Conclusion: The shortcomings of this regulatory framework indirectly benefit companies that outsource ethylene oxide sterilization by omitting essential controls for safety of the patient exposed to possible toxic residues of ethylene oxide, unsafe practices of sterilization of health products, in addition to hindering the control of health service by the country's health surveillance. Keywords: Equipment and supplies. Sterilization. Ethylene oxide; Toxic waste.

RESUMEN: Objetivos: Describir los niveles residuales aceptables de óxido de etileno (OE) en dispositivos médicos, analizar los procesos de aireación recomendados y compararlos con la normativa brasileña. Método: revisión integrativa de la literatura, con descriptores específicos, sin restricción de año de publicación. Búsqueda de datos entre octubre y noviembre de 2019, que resultó en 34 estudios incluidos en el estudio. Resultados: La normativa brasileña actual está desactualizada en cuanto a la clasificación de productos, determinación y valores de residuos tóxicos de OE en dispositivos médicos y procesos recomendados para la aireación de estos productos, lo que puede contribuir al riesgo de eventos adversos para los pacientes que utilizan una aireación inadecuada de dispositivos y, en consecuencia, se necesita urgentemente una actualización. Conclusión: Las brechas en este marco regulatorio benefician indirectamente a las empresas que externalizan la esterilización a OE, al omitir controles esenciales para la seguridad de los pacientes expuestos a posibles residuos tóxicos de OE, favoreciendo prácticas inseguras de esterilización de productos sanitarios, además de dificultar el control de servicio de salud por la Vigilancia Sanitaria del país. Palabras clave: Equipos y suministros. Esterilización. Óxido de etileno; Residuos tóxicos.

'Pós-Doutora em Saúde Pública; Professora Titular da Universidade do Estado da Bahia (UNEB) - Salvador (BA), Brasil. *Autora correspondente: costaeliana2003@hotmail.com

Recebido: 27/02/2020 - Aprovado: 22/07/2021

https://doi.org/10.5327/Z1414-4425202100030008 


\section{INTRODUÇÃO}

A reutilização de dispositivos médicos classificados como "reusáveis" ou de "multiuso" requer a ação de reprocessamento, método que consiste em converter um produto contaminado em um dispositivo pronto para uso e inclui não apenas a limpeza, a desinfecção ou a esterilização do produto, mas também a segurança técnico-funcional, por meio de testes de integridade e funcionalidade ${ }^{1-4}$.

É consenso na literatura que a prática de reprocessamento de produtos para saúde (PPS) é questão complexa, dados os riscos relacionados ao potencial de transmissão de patógenos e os problemas de integridade e desempenho dos produtos reusados ${ }^{5-7}$.

O risco de transmissão de patógenos por meio do uso de produtos médicos depende da presença de microrganismos, do tipo de procedimento a ser realizado e do sítio do corpo onde o produto será inserido 5 . Entre os riscos associados ao reprocessamento e ao reuso de PPS, a literatura cita: infecção, presença de endotoxinas, biofilmes, perda da integridade do material, bioincompatibilidade e outros ${ }^{1-7}$.

Os dispositivos médicos considerados críticos (aqueles que são introduzidos em áreas estéreis do corpo) e termossensíveis (os que não resistem aos métodos de esterilização a altas temperaturas), para serem reutilizados, necessitam de métodos de esterilização a baixa temperatura, a exemplo da esterilização a óxido de etileno (OE), plasma de peróxido de hidrogênio, vapor de formaldeído e ozônio ${ }^{8.9}$. Entre esses métodos, a esterilização a OE é o mais antigo e considerado padrão-ouro, pela alta difusibilidade e potência do gás esterilizante, contudo é também o mais tóxico dos métodos esterilizantes ${ }^{8-19}$.

Os produtos esterilizados pelo OE podem apresentar resíduos tóxicos (etilenocloridrina e etilenoglicol) que, se não removidos, são passíveis de acarretar danos aos pacientes usuários desses produtos, aos profissionais manipuladores e ao meio ambiente. Desse modo, é imperativo que tais PPS sejam submetidos a um processo denominado de "aeração" para remover os resíduos tóxicos ${ }^{8-20}$.

No Brasil, a normativa que regula a esterilização de dispositivos médicos por OE, a Portaria Interministerial no $482^{21}$, data da década de 1990 .

Nesse sentido, este estudo prioriza o risco decorrente dos resíduos tóxicos de $\mathrm{OE}$ em produtos esterilizados por esse agente e procura responder à seguinte questão central: quais são os níveis aceitáveis de resíduos de $\mathrm{OE}$ em dispositivos médicos esterilizados e quais parâmetros norteiam a aeração desses?

\section{OBJETIVOS}

O presente estudo objetiva descrever os níveis residuais aceitáveis de OE em dispositivos médicos, analisar processos de aeração recomendados na literatura e compará-los com a normativa vigente no Brasil, tendo em vista a prevenção de riscos para pacientes usuários desses dispositivos.

\section{MÉTODO}

Trata-se de uma revisão integrativa da literatura, método que permite análises de estudos com distintas metodologias sobre uma temática particular, apresentando potencialidades para o desenvolvimento de novos conhecimentos ${ }^{22}$.

Neste estudo, utilizamos as seguintes etapas: identificação do objeto de estudo e elaboração da questão norteadora, busca de dados e definição dos critérios de inclusão e exclusão da amostra, definição das informações a serem extraídas dos estudos selecionados, avaliação dos estudos incluídos na revisão, apresentação da revisão e síntese dos resultados.

Os estudos foram obtidos por meio de buscas no portal Biblioteca Virtual em Saúde, em periódicos CAPES e em Web of Science. O conjunto de descritores da saúde utilizados na busca de publicações foi: ethylene oxide gas sterilization AND time aeration of sterilized materials; ethylene oxide gas sterilization AND absorption sterilized materials.

Os critérios de inclusão adotados no estudo foram: artigos publicados nos idiomas inglês, espanhol e português, em qualquer ano, nas bases de dados consultadas e com acesso ao texto completo. Excluíram-se artigos sobre $\mathrm{OE}$ que não abordaram a aeração de produtos, publicados em outros idiomas e de acesso por meio de pagamento.

A busca dos dados foi feita online, nos meses de outubro e novembro de 2019, e obtiveram-se 1.189 artigos. Após leitura dos títulos e dos resumos, foram excluídos 827, selecionados 27 artigos e incorporados sete citados nas referências dos estudos selecionados, perfazendo 34 artigos que integram esta revisão (Referências 8 a 19, 20, 21 e 23 a 42).

Após a seleção, foram lidos os artigos disponíveis na íntegra e os resumos dos artigos indisponíveis. Realizou-se a análise dos artigos criticamente, utilizando exploração do material, tratamento dos resultados obtidos, interpretação dos achados, identificando explicações para os resultados diferentes ou conflitantes, objetivando recomendações para a prática. Para sistematizar o estudo, utilizou-se um instrumento de coleta 
de dados que incluiu: nome do artigo, objetivos, método, resultados e conclusão.

Neste artigo, utiliza-se o termo "dispositivo médico" como sinônimo de material, produto médico e PPS, em conformidade com a Agência Nacional de Vigilância Sanitária do Brasil (ANVISA). Usa-se também como sinônimo o termo reprocessamento ou processamento de produtos, a despeito das considerações sobre as diferenças entre esses.

\section{RESULTADOS}

Dos 34 artigos analisados, 13 (38,2\%) são estudos de revisão da literatura, $11(32,3 \%)$ estudos experimentais, oito $(23,5 \%)$ recomendações de órgãos oficiais, dois $(5,8 \%)$ estudos com metodologias variadas. Em relação à origem, 16 estudos (47,0\%) procederam dos Estados Unidos, 11 (32,3\%) do Brasil e sete $(20,5 \%)$ de outros países.

Segue-se a apresentação dos artigos oriundos dessa revisão integrativa.

\section{Apresentação dos estudos selecionados acerca da esterilização a óxido de etileno e aeração de dispositivos médicos}

A esterilização por OE é o método a baixa temperatura mais antigo, utilizado desde 1950, altamente eficaz pelo seu alto poder de penetração, alta difusibilidade e permeabilidade, $\mathrm{o}$ que favorece a esterilização de artigos contidos em embalagens seladas e o acesso a lúmens longos e estreitos compostos de qualquer matéria-prima ${ }^{8-17,23-33,19,34,35}$.

É um agente gasoso explosivo, incolor à temperatura $\mathrm{e}$ à pressão atmosférica, odor desagradável, solúvel em água a $10^{\circ} \mathrm{C}$, em pH ácido reage formando etilenoglicol, reage com o cloro formando etilenocloridrina, ambos tóxicos. Sua excelente atividade bactericida, virucida, fungicida e esporicida é atribuída à alquilação de proteínas e ácidos nucléicos dos

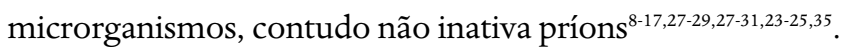

É considerado um agente mutagênico, carcinogênico, teratogênico e neurotóxico. A exposição a esse gás pode causar irritação de olhos, trato respiratório, cefaleia, náusea, vômitos, dermatite de contato, queimaduras por meio de contato direto e, a curto prazo, em altas exposições, pode causar aberrações cromossômicas. Possui efeitos cancerígenos em exposição por inalação, que ocasiona diversos tipos de neoplasias, como leucemia, tumores cerebrais e de mama ${ }^{8-17,27-29,33,35-38,25}$.
O nível máximo de vapores de OE no ar em ambientes laborais é de 1 ppm, numa média de 8 horas de exposição, e de 5 ppm, para o máximo de 15 minutos de exposição ${ }^{8-9,14-17,20,27-28,33,23-24}$.

Com o objetivo de reduzir níveis de OE em locais de trabalho e prevenir o potencial carcinogênico ocupacional relacionado a esse agente, o The US Environmental Protection expediu, em 2010, uma normativa exigindo que hospitais e serviços de saúde utilizem câmara única, que combine esterilização e aeração ${ }^{34,39}$.

A esterilização a $\mathrm{OE}$ requer controles especiais que são inter-relacionados, variam com o tipo do esterilizador, mas, de modo geral: concentração do gás de 450 a $1.200 \mathrm{mg} / \mathrm{L}$; temperatura entre 37 e $63^{\circ} \mathrm{C}$; umidade relativa de 40 a $80 \%$ e tempo de exposição de 4 a 6 horas. O monitoramento deve ser realizado por meio de monitores mecânicos (temperatura, concentração do gás, pressão, tempo de exposição) em cada carga de esterilização, monitores químicos (cada pacote) e monitores biológicos (Bacillus atrophaeus sp) em cada ciclo. O ciclo básico consiste em cinco estágios: pré-condicionamento ou umidificação, introdução do gás, exposição, evacuação e irrigação e lavagem do ar ${ }^{8-9,14-17,19-20,29,33,35-37}$.

Níveis excessivos de OE residual ou de seus produtos de degradação, tais como etilenoglicol e etilenocloridrina, em produtos médicos são potencialmente tóxicos e devem ser removidos dos materiais após o ciclo de esterilização para prevenir danos aos pacientes usuários desses produtos $^{8-9,14-20,29-33-36,40,26}$.

A aeração pode ser realizada pela própria câmara do esterilizador (aeração mecânica) ou em salas exclusivas (aeração ambiental). A aeração mecânica é considerada o método mais eficiente e seguro de remoção de resíduos de $\mathrm{OE}^{34-35,39-41}$.

A aeração ambiental deve ser realizada em salas exclusivas, de uso restrito, sob pressão negativa, com controle de temperatura do ar e do fluxo (mínimo de 25 trocas de ar/ hora) $)^{21,31,33,25}$ e exaustão para o exterior do ambiente. Esse processo de aeração, além de longo, expõe os trabalhadores a vapores tóxicos durante a transferência das cargas esterilizadas para a sala de aeração, pode não ser efetivo, a depender das condições de temperatura e fluxo de ar, e é contraindicado por órgãos reguladores internacionais ${ }^{20,27,34-35,39}$.

A duração da aeração depende de algumas variáveis, tais como: propriedade do dispositivo médico (composição, forma, densidade, peso e embalagem utilizada), tipo de embalagem, tipo do esterilizador, temperatura da câmara de aeração, número de trocas do filtro de ar por hora e características do fluxo de ar, bem como da intenção de uso do artigo (uso externo ou interno $)^{8-11,16-17,20,30,40,26,35}$. 
Parâmetros da aeração mecânica também influenciam a duração do tempo requerido para remover $\mathrm{OE}$ dos produtos, e a temperatura e os números de troca de ar da câmara têm impacto significante no processo de aeração. Quanto maior a temperatura, menor o tempo requerido para aeração de produtos ${ }^{16-17,20,30,40,35}$.

Autores referem que o tempo de aeração, a depender da composição, do tamanho dos dispositivos, do sistema de aeração e do tipo do esterilizador, pode variar de seis horas a sete dias ${ }^{29,26}$, e outro autor ${ }^{33}$ recomenda tempo mínimo de 12 horas de aeração, quando na ausência de especificação pelo fabricante do produto.

Estudo cubano aponta que a eliminação total ou a diminuição de resíduos de $\mathrm{OE}$ a limites permissíveis requer um processo de destoxificação, que pode variar de 48 horas a dez dias ou mais, na dependência do número de esterilizações realizadas no mesmo dispositivo ${ }^{36}$.

Antes da normativa que proibiu a aeração ambiental em serviços de saúde nos Estados Unidos ${ }^{39}$, o Center for Disease Control and Prevention (CDC) recomendava aeração mecânica durante oito a 12 horas à temperatura de $50 \mathrm{a} 60^{\circ} \mathrm{C}$ ou aeração ambiental em salas exclusivas durante sete dias a $20^{\circ} \mathrm{C}^{8-9,27}$.

Em Cuba, a aeração de produtos esterilizados em OE segue um padrão rigoroso, com tempo de aeração dentro da câmara de 24 horas após o término da esterilização, tempo de detoxificação a $60^{\circ} \mathrm{C}$ durante oito horas e tempo de aeração ambiente em local ventilado de sete a dez dias ${ }^{15}$.

Estudo brasileiro objetivou determinar resíduos de OE em frascos enterais de polietileno esterilizados por esse agente. Após ciclo padrão de esterilização $(430 \mathrm{mg} / \mathrm{L}$ de OE, durante 6 horas, a $55^{\circ} \mathrm{C}$ e umidade relativa $>35 \%$ ), as amostras foram aeradas mecanicamente com 14 pulsos de ar e vácuo e colocadas em sala de aeração ambiental com temperatura de $55^{\circ} \mathrm{C}$ e 27 trocas de ar/hora. As amostras foram retiradas nos tempos 0, 4, 12, 24 e 48 horas, e a concentração residual das amostras analisadas para o limite de $10 \mu \mathrm{g} / \mathrm{mL}$ de $\mathrm{OE}$ demonstrou significativa redução residual $(\mathrm{p}<0,05)$ a partir do tempo de 6 horas de aeração ${ }^{31}$.

Outro estudo que determinou o tempo de aeração em produtos esterilizados a $\mathrm{OE}$ e embalados em containers rígidos revelou a necessidade de 17 horas de aeração para a isenção de resíduos desse agente ${ }^{32}$.

Pesquisa que avaliou o tempo de aeração de produtos esterilizados por OE na Região Sudeste do Brasil identificou que $100 \%$ das empresas esterilizadoras a OE possuem a sala de aeração preconizada pela norma brasileira, mas apresentam parâmetros heterogêneos em relação à aeração ambiental ${ }^{14}$.

A norma internacional American National Standards Institute/Association for the Advancement of Medical Instrumentation/International Organization for Standardization (ANSI / AAMI/ ISO) 10993- $7^{20}$ atualizou em 2012 os níveis residuais aceitáveis de OE e etilenocloridrina (ETCH) para dispositivos médicos. Essa normativa utiliza como critério a duração do tempo de contato do produto com o paciente e, para tanto, classifica-a como "exposição limitada" (tempo <24 horas), "exposição prolongada" (>24 horas e não excede 30 dias) e "exposição permanente" (>30 dias). A dose diária residual de $\mathrm{OE}$ e ETCH não deve ultrapassar $20 \mathrm{mg}$ e $12 \mathrm{mg}$, respectivamente, e, para os produtos de exposição prolongada e permanente, não deve ultrapassar o limite de $60 \mathrm{mg}$, conforme Quadros 1 e 2 .

As recomendações da Portaria Interministerial no 482/1999 11 diferem das normas e dos estudos internacionais em vários controles, especificamente em relação aos níveis aceitáveis de resíduos de OE, bem como dos processos recomendados para a aeração de PPS.

A normativa brasileira classifica os produtos implantáveis por peso (10 a $100 \mathrm{~g}$ ) e segundo contato do dispositivo com mucosa, sangue e pele do paciente e preconiza controle de resíduos de $\mathrm{EO}$ em produtos disponibilizados para pronto uso e considerados como de uso único, a exemplo de "esponjas cirúrgicas", "dispositivos intrauterinos" e "lentes intraoculares".

Na Portaria $n^{\circ} 482$ / 1999, os níveis residuais de OE e ETCH variam de $25 \mathrm{ppm}$ (correlatos que contatam sangue) a $250 \mathrm{ppm}$ (correlatos que contatam mucosa), limites esses determinados pelo Food and Drug Administration (FDA) em $1978^{42}$ e em desacordo com os níveis atuais para esses resíduos tóxicos, conforme descrito no Quadro 3.

Quadro 1. Limites máximos aceitáveis de resíduos de óxido de etileno e etilenocloridrina em produtos para saúde, segundo tempo de contato.

\begin{tabular}{|l|l|l|}
\hline Tempo de contato & \multicolumn{1}{|c|}{ Óxido de etileno } & \multicolumn{1}{c}{ Etilenocloridrina } \\
\hline Produto médico com contato prolongado & $\begin{array}{l}\text { Máximo de } 4 \mathrm{mg} \text { nas primeiras } 24 \text { horas. } \\
\text { Máximo de } 60 \mathrm{mg} \text { nos primeiros } 30 \text { dias. }\end{array}$ & $\begin{array}{l}\text { Máximo de } 9 \text { mg nas primeiras } 24 \text { horas. } \\
\text { Máximo de } 60 \text { mg nos primeiros } 30 \text { dias. }\end{array}$ \\
\hline Produto médico com contato permanente & $\begin{array}{l}\text { Máximo de } 4 \mathrm{mg} \text { nas primeiras } 24 \text { horas. } \\
\text { Máximo de } 60 \mathrm{mg} \text { nos primeiros } 30 \text { dias. }\end{array}$ & Máximo de 9 mg nas primeiras 24 horas. \\
\hline
\end{tabular}

Fonte: ANSI/AAMI/ISO 10993-7:2008 (R) 2012 
Quanto aos processos de aeração de produtos, a norma vigente no Brasil apenas recomenda uma sala de aeração para os serviços que realizam a esterilização por esse método, sem mencionar a aeração mecânica nem definir o tempo de exposição para a aeração ambiental.

\section{Síntese dos resultados}

Os resultados desta revisão apontam o OE como agente esterilizante ideal para dispositivos críticos e sensíveis ao calor, a despeito da toxicidade e da obrigatoriedade da realização de controles relacionados aos esterilizadores, trabalhadores, dispositivos médicos e meio ambiente.

Como exposto, o marco regulatório brasileiro está desatualizado em relação à determinação de resíduos tóxicos de OE de produtos médicos, contribuindo para o risco sanitário relacionado a esse método de esterilização.

Inicialmente, a classificação de produtos segundo "contato com mucosa, sangue e pele do paciente", adotada nessa legislação, destoa da atual norma internacional ${ }^{20}$, que especifica limites máximos segundo o risco toxicológico desses resíduos para o paciente, considerando a duração do tempo em que o paciente é exposto ao dispositivo esterilizado com $\mathrm{OE}$.

Os valores dos níveis residuais definidos na Portaria $\mathrm{n}^{\circ}$ 482 são maiores do que os recomendados na norma internacional, aumentando o risco potencial de eventos adversos para pacientes usuários de produtos reprocessados por OE.

A norma brasileira preconiza o processo de aeração ambiental para a dessorção dos produtos esterilizados por OE, processo esse que pode não ser efetivo, pois depende de muitas variáveis. Ademais, é contraindicado, por estar proscrito nos Estados Unidos desde $2010^{20,39}$. Nesse sentido, a aeração mecânica, não mencionada por essa norma, constitui o padrão-ouro da aeração de produtos esterilizados por OE, contudo esse processo também requer controles de temperatura e fluxos de ar dentro da câmara

Há consenso entre autores de que a duração da aeração depende de fatores já descritos nesta revisão e, entre esses produtos confeccionados à base de cloreto de polivinil (PVC), poliestireno e borrachas são os que mais absorvem OE. Assim, não existe um tempo padrão recomendado para aeração de todos os dispositivos esterilizados por esse agente $e^{8-11,16-17,20,30,40,23,26,18,35}$.

Objetivando superar as dificuldades relacionadas à duração da aeração dos PPS, um estudo recomenda que, se a composição do dispositivo médico for desconhecida, a aeração deve ser realizada como no maior desafio (como se o produto fosse de PVC), utilizando aeração mecânica a $50^{\circ} \mathrm{C}$ por 12

Quadro 3. Limites máximos de resíduos de óxido de etileno em correlatos (ppm).

\begin{tabular}{|l|c|c|c|}
\hline Correlato & OE & ETCH & ETG \\
\hline Implantes & & & \\
\hline Pequeno $(10 \mathrm{~g})$ & 250 & 250 & 5.000 \\
\hline Médio $(>10-<100 \mathrm{~g})$ & 100 & 100 & 2.000 \\
\hline Grande $(>100 \mathrm{~g})$ & 25 & 25 & 500 \\
\hline Dispositivos intrauterinos & 5 & 10 & 10 \\
\hline Lentes intraoculares & 25 & 25 & 500 \\
\hline Correlatos que contatam a mucosa & 250 & 250 & 5.000 \\
\hline Correlatos que contatam o sangue & 25 & 25 & 250 \\
\hline Correlatos que contatam a pele & 250 & 250 & 5.000 \\
\hline Esponjas cirúrgicas & 25 & 250 & 500 \\
\hline
\end{tabular}

OE: óxido de etileno; ETCH: etilienocloridrina; ETG: etilenoglicol.

Fonte: Brasil. Portaria Interministerial n. 482/1999.

Quadro 2. Sumário dos limites permitidos para óxido de etileno e etilenocloridrina (limites por produto).

\begin{tabular}{|c|c|c|}
\hline Categoria do produto para saúde & Óxido de etileno & Etilenocloridrina \\
\hline Produto com contato limitado (<24 horas) & $4 \mathrm{mg}$ & $9 \mathrm{mg}$ \\
\hline Produto com contato prolongado ( $>24 \mathrm{~h}<30$ dias) & $60 \mathrm{mg} / 30 \mathrm{dias}$ & $60 \mathrm{mg} / 30 \mathrm{dias}$ \\
\hline Produto com contato permanente ( $>30$ dias) & $2,5 \mathrm{~g} / \mathrm{por}$ toda a vida & $10 \mathrm{~g} /$ por toda a vida \\
\hline Limite de contato tolerável & $10 \mu \mathrm{g} / \mathrm{cm}^{2}$ & $5 \mathrm{mg} / \mathrm{cm}^{2}$ \\
\hline Lentes intraoculares & $0,5 \mu \mathrm{g} /$ lente/dia $1,25 \mu \mathrm{g} /$ lente & $4 \times$ limite sugerido para $\mathrm{OE}$ \\
\hline Separadores de células sanguíneas (aferese) & $10 \mathrm{mg}$ & $22 \mathrm{mg}$ \\
\hline Oxigenadores de sangue & $60 \mathrm{mg}$ & $45 \mathrm{mg}$ \\
\hline Produtos de Bypass cardiopulmonar & $20 \mathrm{mg}$ & $9 \mathrm{mg}$ \\
\hline Produtos de purificação do sangue $=-0987654321+$ (hemodialisadores) & $4,6 \mathrm{mg}$ & $4,6 \mathrm{mg}$ \\
\hline Curativos em contato com pele íntegra & $10 \mu \mathrm{g} / \mathrm{cm}^{2}$ & $5 \mathrm{mg} / \mathrm{cm}^{2}$ \\
\hline
\end{tabular}

Fonte: ANSI/AAMI/ISO 10993-7:2008 (R) 2012. 
horas ou a $60^{\circ} \mathrm{C}$ por oito horas, e a The American Hospital Association recomenda esses parâmetros como o mínimo para qualquer tipo de dispositivo médico ${ }^{35}$.

Tendo em vista que a maioria dos produtos encaminhados para esterilização a OE são termossensíveis e constituídos de polímeros plásticos, muitos de PVC, a possibilidade de absorção de altos volumes tóxicos residuais aumenta à medida que são submetidos a repetidas esterilizações ${ }^{35}$, situação comum na rotina dos centros de material e esterilização (CME) brasileiros, demandando, no mínimo, conhecimento acerca dos níveis residuais de OE e ETCH dos produtos reprocessados.

Ocorre que a normativa brasileira não regulamenta a periodicidade dos controles de resíduos de OE nos produtos esterilizados pelas empresas que terceirizam o processo de esterilização a OE. Consequentemente a realização e o envio desses controles para os serviços de saúde contratantes dependem da disponibilidade, da iniciativa e do interesse dessas empresas, ficando os serviços de saúde reféns desse contexto.

Tendo em vista as considerações anteriormente descritas e a problemática que envolve a aeração de produtos esterilizados a OE, ratificamos alguns questionamentos suscitados por autora $^{35}$ ainda na década de 1990 , ao tempo que incluímos outras questões que nos parecem também pertinentes e atuais: quais são os critérios utilizados pelos enfermeiros gerentes dos CME para indicar esterilização a OE para determinados produtos? A rotina de envio e recebimento dos produtos esterilizados a OE é compatível com seu processo de aeração? Qual é a periodicidade de recebimento de laudos acerca dos níveis residuais de $\mathrm{OE}$ dos produtos esterilizados por esse agente? Tendo em vista que o volume de absorção do OE depende também do número de esterilizações, qual é a frequência da utilização desses produtos? Os líderes da assistência à saúde têm conhecimento acerca das implicações relacionadas à esterilização pelo OE?

\section{CONCLUSÃO}

Este estudo alcançou seus objetivos ao descrever os níveis residuais de $\mathrm{OE}$ em produtos esterilizados por esse agente, os métodos de aeração desses produtos e ao comparar a normativa brasileira vigente com estudos internacionais sobre essa temática.

Consideramos como possível limitação o fato de que este estudo teve como suporte teórico artigos de revisão e recomendações de órgãos oficiais, com pequena parte constituída de estudos experimentais, que poderiam reportar os riscos relacionados à toxicidade do $\mathrm{OE}$, de modo que contextualizassem situações mais concretas.

Evidenciou-se que a Portaria $n^{\circ} 482$ está desatualizada em pontos cruciais de controle e segurança da esterilização de dispositivos médicos esterilizados a $\mathrm{OE}$, a exemplo da obsolescência dos níveis aceitáveis de resíduos desse agente, classificação de produtos e métodos de aeração, contribuindo para a ocorrência de riscos de eventos adversos para pacientes, trabalhadores e meio ambiente e, nesse sentido, urge uma atualização dessa normativa em vigor.

Ademais, as lacunas desse marco regulatório beneficiam indiretamente as empresas que terceirizam esterilização a $\mathrm{OE}$ ao omitir os controles essenciais para segurança do paciente, usuário de produtos esterilizados por esse agente extremamente tóxico, favorecer práticas inseguras de esterilização de produtos médicos, além de dificultar o controle sanitário de serviço de saúde pelas vigilâncias sanitárias do país.

\section{REFERÊNCIAS}

1. Mansur JM. Reuse of single use devices: understanding risks and strategies for decision-making for healthcare organizations. Joint Comission International [Internet]. 2017 [acessado em 26 dez. 2019]. Disponível em: http://www.jointcomissioninternational.org

2. Jacobs $P$, Akpinar I. Single use medical devices: economics issues. Heart Asia. 2018;10(2):e011034. http://doi.org/10.1136/ heartasia-2018-011034

3. Unger S, Landis A. Assessing the environmental, human health, and economic impacts of reprocessed medical devices in a Phoenix hospital's supply chain. J Clean Prod. 2016;112:1995-2003. http:// doi.org/10.1016/j.jclepro.2015.07.144
4. Kapoor A, Vora A, Nataraj G, Mishra S, Kerka P, Manjunath CN. Guidance on reuse of cardio-vascular catheters and devices in India: a consensus document. India Heart J. 2017;69(3):357-63. http://doi. org/10.1016/j.ihj.2017.04.003

5. World Health Organization. Global Guidelines for The Prevention of Surgical Site Infection. Geneva. WHO [Internet]. 2016 [acessado em 02 dez. 2019]. Disponível em: https://apps.who.int/iris/ handle/10665/277399

6. Costa EAM, Santos QNF, Dantas IS. Risco em processamento de produtos para saúde em unidade básicas de Salvador, BA. Vigil Sani Debate. 2018;6(2):38-43. http://doi.org/10.22239/2317-269x.00991 
7. Costa EAM. Panorama internacional do reprocessamento de produtos para saúde de uso único. Rev SOBECC. 2016;21(4):203-9. http://doi. org/10.5327/Z1414-44252016000400005

8. Rutala WA, Webwe DJ. Sterilization, high-level disinfection an environmental cleaning. Infect Dis Clin N Am. 2011;25(1):45-76. http://doi.org/10.1016/j.idc2010.11.009

9. Rutala WA, Weber DJ. Healthcare Infection Control Practices Advisory Committee. Guidelines for Disinfection and Sterilization in Helathcare Facilities. Center for Disease Control and Prevention. HICPAC [Internet]. 2008 [acessado em 22 jan. 2020]. Disponível em: https://www.cdc.gov/infectioncontrol/pdf/guidelines/disinfectionguidelines-H.pdf

10. Goveia VR, Pinheiro SMC, Graziano KU. Métodos de esterilização por baixa temperatura e novas tecnologias. Rev Latino-am Enferm. 2007;15(3). http://doi.org/10.1590/S0104-11692007000300002

11. Souza RQ, Graziano KU, Ikeda TI, Gonçalves CR, Cruz AS. Citotoxicidade de tubos de PVC esterilizados em óxido de etileno após exposição à radiação gama. Rev Esc Enferm USP. 2013;47(2):494-9. http://doi. org/10.1590/S0080-62342013000200031

12. Abdo N, Rodrigues DB, Freitas MIP. Métodos físico-químicos de esterilização. Óxido de Etileno. In: Associação Paulista de Epidemiologia e Controle de Infecção Relacionada à Assistência à Saúde (APECIH). Limpeza, Desinfecção e Esterilização de Artigos em Serviços de Saúde. São Paulo: APECIH; 2010.

13. Sundim S. Riscos na reutilização de produtos médicos esterilizados em óxido de etileno [dissertação]. Mestrado em Engenharia Biomédica. Curitiba: Universidade Tecnológica Federal do Paraná; 2014.

14. Abdo N. Aeração de artigos odonto-médico-hospitalares reprocessados a óxido de etileno: a prática em empresas prestadoras de serviço terceirizado [dissertação]. Campinas: Faculdade de Ciências Médicas. Universidade Estadual de Campinas; 2006.

15. Rodrigues RH, Fernández VMC, Despaigne SC, Betacourt OV. Dispositivos médicos de uso único reprocesados por esterilización química mediante óxido de etileno. Rev Cubana Hig Epidemiol [Internet]. 2002;40(2):89-94 [acessado em 30 jan. 2020]. Disponível em: http://scielo.sld.cu/pdf/hie/v40n2/hie02202.pdf

16. Shintani $H$. Ethylene oxide gas sterilization on medical devices. Biocontrol Sci. 2017;22(1):1-16. http://doi.org/10.4265/bio.22.1

17. Lucas AD, Forrey C, Saylor DM, Vorvolakos K. Solvent on thermal extraction of ethylene oxide from polymeric materials: medical device considerations. J Biomed Mater Res Appl Biomater. 2018;106(6):245563. http://doi.org/10.1002/jbm.b.34052

18. Lucas AD, Merritt K, Hitchins VM, Woods TO, McNamec SG, Lyle DB, et al. Residual ethylene oxide in medical devices and device material. J Biomed Mater Res Part B: Appl Biomater. 2003;66B:548-52. http:// doi.org/10.1002/jbm.b.10036

19. Mendes GCC, Brandão RS, Silva CLM. Ethylene oxide sterilization of medical devices: a review. Am J Infect Control. 2007;35:574-81. http://doi.org/10.1016/j.ajic.2006.10.014

20. ANSI/AAMI/ ISO 10993-7:2008(R)2012. Biological evaluation of medical devices - Part 7. Ethylene oxide sterlization residuals. AAMI: Arlington; 2012.
21. Brasil. Portaria Interministerial n. 482, de 16 de abril de 1999. Aprova Regulamento Técnico contendo disposições sobre os procedimentos de instalações de Unidade de Esterilização por Óxido de Etileno e de suas misturas e seu uso [Internet]. Brasília (DF): Ministério da Saúde e Ministério do Trabalho e Emprego; 1999 [acessado em 30 jan. 2020]. Disponível em: https://bvsms.saude.gov.br/bvs/saudelegis/ gm/1999/pri0482_16_04_1999.html

22. Mendes KDS, Silveira RCCP, Galvão CM. Revisão integrativa: método de pesquisa para a incorporação de evidências na saúde e na enfermagem. Texto Contexto Enferm. 2008;17(4):758-64. https:// doi.org/10.1590/S0104-07072008000400018

23. Gimeno P, Auguste ML, Handlos V, Nielson AM, Schmidt S, Lassu N, et al. Identification and quantification of ethylene oxide in sterilized medical devices using multiple headspace GC/MS measurement. JPharm Biomed Anal. 2018;119-27. http://doi.org/10.1016/jpba.2018.05.035

24. El Helaly M, Balkhy H, Vallenius L, Khan W. Occupational exposure and work practices among ethylene oxide sterilization workers. Egypt J Occup Med. 2017;41(2):157-74. http://doi.org/10.21608/ EJOM.2017.2849

25. Luttrell W, Brotherton EE. Ethylene oxide. J Chem Health Saf. 2008;15(6):30-2 http://doi.org/10.1016/j.jchas.2008.09.010

26. Gibson C, Mattheus IP, Sanuel AH. Computerized model for accurate determination of ethylene oxide diffusion in sterilized medical supplies. Biomaterials. 1989;10(5):343-48. http://doi.org/10.1016/ 0142-9612(89)90076-8

27. Center for Disease Control and Prevention. The National Institute for Occupational Safety and Health (NIOSH). Ethylene oxide sterilizers in health care facilities: engeneering controls and workpractices [Internet]. Atlanta: NIOSH; 1989 [acessado em 03 jan. 2020]. Disponível em: https://www.cdc.gov/niosh/docs/89-115/default.html

28. Bogen KT, Sheehan PJ, Valdez Flores C, Li AA. Reevaluation of historical exposures to ethylene oxide among US sterilization workers in The National Institute of Occupational Safety and Health (NIOSH) study cohort. Int J Environ Res Public Health. 2019;16(10):1738. https:// doi.org/10.3390/ijerph16101738

29. Andrade Júnior FP, Romano TKF, Lima BTM, Souza EKM, Alves TWB, Silva IB, et al. Óxido de etileno como agente esterilizante: características gerais, toxicidade e comparação com outros saneantes e métodos de esterilização. J Tche Chem. 2019;16(31):175-85.

30. Mendes GC, Brandão TRS, Silva CLM. Kinetics of ethylene oxide desorption from sterilized materials. J AOAC Int. 2013;96(1):33-6. http://doi.org/10.5740/jaocint.11-506

31. Santos HCO. Validação do processo de esterilização por óxido de etileno para determinar tempo de aeração em frascos enterais [dissertação]. Goiânia: Faculdade de Farmácia. Universidade Federal do Goiás; 2010.

32. Nakata S, Umeshita K, Ueyama H, Takashina M, Noguchi S, Murata A, et al. Aeration time following ethylene oxide sterilization for reusable rigid sterilization containers: concentration of gaseous ethylene oxide in containers. Biomed Instrum Technol. 2003;34(2):121-4. PMID: 10820640

33. Anaya LPL. Óxido de etileno, utilización como agente esterilizante y riesgos para la salud del personal sanitário. CES Public Health. 2014;5(2):154-62. http://doi.org/10.21615/3019 
34. Boiano JM, Steege AL. Ethylene oxide and hidrogen peroxide gas plasma setrilization: precautionary practices in US Hopsitals. Zentralsterilisation (Wiesb). 2015;23(4):262-8. PMID:26594097

35. Steelman V M. Ethylene oxide. The importance of aeration. AORN J. 1992;55(3):773-87. http://doi.org/10.1016/s0001-2092(07)69447-2

36. Rodriguez RH, Fernández CG, Despaigne SC. Análisis cuantitativo y efecto de resíduos em processos esterilización química a bajas temperaturas. Rev Cubana Invest Biomed [Internet]. 2006;25(2) [acessado em 30 jan. 2020]. Disponível em: http://scielo.sld.cu/scielo. php?script=sci_abstract\&pid=S0864-03002006000200002\&lng=en \&nrm=iso\&tlng=en

37. Hernádez-Navarrete MJ, Celorrio-Pascual JM, Moros CL, Bermad VMS. Fundamentos de antissepsia, desinfeccion y esterilización. Enfem Infec Microbiol Clin. 2014;32(10):681-8. http://doi.org/10.1016/j.eimc.2014.04.003

38. Savaris M, Carvalho GA, Falavigna A, Santos V, Brandalise RN Avaliação química e térmica da esterilização PEEK comercial e médica por óxido de etileno. Esteira. Res. 2016;19(4).
39. US Environmental Protection Agency. Ethylene oxide: hospitals and healthcare facilities must use a single chamber where sterilizing medical equipment with ethylene oxide [Internet]. 2010 [acessado em 13 feb. 2020]. Disponivel em: https://archive.epa.gov/pesticides/ reregistration/web/html/ethylene_oxide_fs.html

40. Dias FN, Ishin M, Nogaroto SL, Piccini B, Penna TCV. Sterilization of medical devices by ethylene oxide, determination of the dissipation of residues and use of green fluorescent protein as an indicator of process control. J Biomed Mater Res. 2009;91B(2):629-30. http:// doi.org/10.1002/jbm.b.31438

41. Bogen KT, Sheehan PJ, Valdez-Flores C, Li AA. Reevaluation of historical exposures to ethylene oxide among US sterilization workers in The National Institute of Occupational Safety and Health (NIOSH) study cohort. Int J Environ Res Public Healthy. 2019;16(10):1738. http://doi.org/10.3390/ijerph16101738

42. US Food and Drug Administration. Federal Register [Internet]. 1987 [acessado em 13 fev. 2020]. Disponível em: https://www.govinfo. gov/app/details/FR-1978-06-23 